International Journal of Wireless \& Mobile Networks (IJWMN) Vol. 3, No. 3, June 2011

\title{
POWER EFFICIENT CLUSTERING PROTOCOL (PECP)- HETEREGENOUS WIRELESS SENSOR NETWORK
}

\author{
S.Taruna ${ }^{1}$, Kusum Jain ${ }^{2}$, G.N. Purohit ${ }^{3}$ \\ Department of Computer Science, Banasthali University, Rajasthan, India \\ ${ }^{1}$ staruna 71 @yahoo. com \\ ${ }^{2}$ Kusum_20000rediffmail.com \\ ${ }^{3}$ gn_purohitjpeyahoo.co.in
}

\begin{abstract}
In this paper we propose a new Power Efficient Clustering Protocol(PECP) for prolonging the sensor network lifetime. Homogeneous clustering protocols assume that all the sensor nodes are equipped with the same amount of energy and as a result, they cannot take the advantage of the presence of node heterogeneity. Adapting this approach, we propose a new protocol named PECP (Power Efficient Clustering Protocol) to improve the stable region of the clustering hierarchy process using the characteristics parameters of heterogeneity, namely the fraction of powerful nodes( with more energy) more suitable to become a cluster head. Intuitively, powerful nodes have to become cluster heads more often than normal nodes. The performance of the PECP via computer simulation is evaluated and compared with other clustering algorithms. It has been found that PECP prolongs the sensor network lifetime.
\end{abstract}

\section{KEYWORDS}

Clustering Protocols, homogeneous, Network Lifetime, Cluster head, heterogeneity.

\section{INTRODUCTION}

A sensor network consists of a large number of very small nodes that are deployed in some geographical area. These tiny sensor nodes, which consist of sensing, data processing, and communicating components, leverage the idea of wireless sensor networks [1], [2]. As sensor nodes may be placed everywhere, this type of network can be applied to multiple scenarios [3]. e.g., in healthcare [4], where they are used to monitor and assist disabled patients, habitat monitoring [5], disaster management [6], and even for commercial applications such as managing an inventory, monitoring product quality, surveillance, and target tracking [7].

In cluster based architectures, mobile nodes are divided into virtual groups. Each cluster has adjacencies with other clusters. All the clusters have the same rules. A cluster can be made up of a Cluster Head node and Cluster Members [8]. In this kind of network, Cluster Head nodes are used to control the cluster and the size of the cluster is usually about one or two hops from the Cluster Head node. There are many cluster based architectures [9]. Sensor networks clustering schemes can be classified according to several criteria. For example, they can be classified according to whether the architectures are based on Cluster Head [10] or on Non Cluster Head [11]. The first architecture needs a Cluster Head to control and manage the group, and the second one does not have a specific node to perform this task. Another way to differentiate the cluster-based architectures is observing the hop distance between node pairs in a cluster.

The concept of cluster based routing is also utilized to perform energy efficient routing in WSNs. Author[12] shows the most important features of cluster-based architectures over ad hoc

DOI : 10.5121/ijwmn.2011.3305 
and sensor networks. The last feature is strongly linked with energy conservation, given that clustered wireless sensor networks offer two major advantages over their non-clustered counterparts; firstly, clustered wireless sensor networks are capable of reducing the volume of inter-node communication by localizing data transmission within the formed clusters and decreasing the number of transmissions to the sink node; secondly, clustered wireless sensor networks are capable of extending the nodes' sleep times by allowing cluster heads to coordinate and optimize the activities. Some of routing protocols in this group are: LEACH (13), PEGASIS (14).

Researchers generally assume that the nodes in wireless sensor networks are homogeneous, but in reality, homogeneous sensor networks hardly exist. In heterogeneous sensor networks, typically, a large number of inexpensive nodes perform sensing, while a few nodes having comparatively more energy to perform data filtering, fusion and transport. This leads to the research on heterogeneous networks where two or more types of nodes are considered. Heterogeneity in wireless sensor networks can be used to prolong the lifetime and reliability of the network.

For heterogeneous WSNs, a very critical task for clustering protocols is to select the cluster head so that least energy is consumed, and hence prolong the lifetime. Clustering algorithms can be classified based on two main criterions: according to the energy efficiency and stability. Selection of cluster head in energy efficient techniques generally depends on the initial energy, residual energy, average energy of the network, or energy consumption rate or combination of these. The stable election protocols for clustered HWSN prolong the time interval before the death of first node called stability period. Many of the algorithms are proposed for clustering in heterogeneous network. Following are the some algorithm for clustering: EEHC[15], DEEC [16], DBEC 17] and CBSD [18].

The main contribution of our work is the design and verification of a new clustering protocol for heterogeneous sensor network and its comparison with other protocols in existence. In this paper, we present a proposal for new cluster head selection. The proposed algorithm is zone based algorithm and heterogeneity parameter is energy. In this algorithm we reduce the number of communication between the sensors nodes for cluster head selection, so that the energy consumption for cluster head selection can be further reduce.

Rest of the paper is organized as follows. Section 2 formulates the problem, explains which issue has to be solved and presents some application environments where our proposal can be used. Section 3 describes our proposal. Its scalability is demonstrated in Section 4.Section 5 describe the energy model. Section 6 gives the proposed algorithm and operation. The comparison of the proposed protocol with other existing cluster based protocol is shown in Section-7. Finally, Section 8 gives the conclusion.

\section{Problem Formulation and ApPlication Environments}

Wireless sensor network generally have the architecture presented in [1]. Let us suppose an environment where a great variety of sensors must be scattered to take measurements from the environment. Let us also suppose that the area is divided into rectangular zones. The network has two types of nodes: Cluster Head $(\mathrm{CH})$ and cluster member $(\mathrm{CM})$. The $\mathrm{CM}$ senses the data and send to $\mathrm{CH}$ in specific time duration or when an event happens in the network. $\mathrm{CH}$ is responsible to send data to Base Station (BS). BS is situated far and out of network. $\mathrm{CH}$ is also responsible for data aggregation and data compression that reduces the energy consumed for data transmission from $\mathrm{CH}$ to $\mathrm{BS}$.

Following type of communication will take place in the network:-

1. CM senses the data from the environment and sends it to $\mathrm{CH}$.

2. $\mathrm{CH}$ aggregate the data come from $\mathrm{CM}$ and compress the data and send it to BS. 
3. Control information from $\mathrm{BS}$ to $\mathrm{CM}$ and $\mathrm{CH}$.

4. Control information from $\mathrm{CM}$ and $\mathrm{CH}$ to $\mathrm{BS}$.

Taking into account the aforementioned premises, several application environments can be found. Some of them are the following:

1. Applications with the fixed deployment area. In which the area can be divided in rectangular grid. For example like WSN for fire detection. It gives the good results work with applications in which node are fixed. For example WSN for fire detection.

2. Application areas where the movement of node is less or symmetric. In this the means of symmetric is: the density of zone should not be so much affect by the movement of node.

3. It could be used in any kind of system where an event or alarm is based on what is happening in a particular zone of network. One example is a group-based system to measure the environmental impact of a place (forest, marine reef, etc.).

4. Agricultural application for example some harvests like saffron: a small change in the temperature can be damage the plants or flower of the saffron.

5. WSN for Traffic Management.

\section{Architecture Proposal Description}

From the logical point of view, let us suppose an environment where a large number of same type of sensors are scattered to take measurements from the environment. The area is divided into rectangular grid called zones and each zone has several sensors sensing the environmental value like humidity, temperature, wind, movement, etc., organized by a central node as a cluster head $(\mathrm{CH})$. The network has 2 type of node cluster heads $(\mathrm{CHs})$ and Cluster members ( $\mathrm{CMs}$ ). Each cluster head have an association with some sensing node called Cluster Members (CMs). Although CHs have sensing capacities, they are the ones with higher capabilities then CMs. $\mathrm{CMs}$ only sense the environment and send the sense data to the $\mathrm{CH}$. The same physical and MAC layers are used between CHs and their CMs. CHs organize and control the CMs in their cluster and all CMs have to establish a connection with a $\mathrm{CH}$ to join its cluster. This connection can be established only if the distance between them is shorter than other CHs in the network. All $\mathrm{CHs}$ and $\mathrm{CMs}$ can communicate with the BS. In our design only one $\mathrm{CH}$ per cluster has been provided, but more could be added for scalability purposes, using the algorithm presented by the same authors of this paper in reference [25]. An example of architecture proposed is shown in figure 1 . The area is geographically divided in zone.

\begin{tabular}{|c|c|c|}
\hline 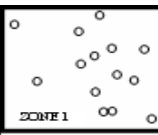 & 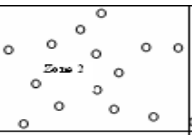 & 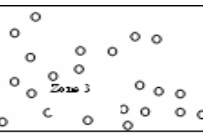 \\
\hline 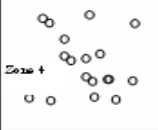 & $\begin{array}{ccccc}0 & 0 & 0 & 0 \\
0 & 0 & 0 & 0 \\
0 & 0 & \text { 50ास } & 0 \\
0 & 0 & 0 & 0\end{array}$ & 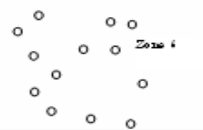 \\
\hline
\end{tabular}

Figure 1: wireless sensor network

Many of the routing protocol can be used to route information between CHs. For Example OLSR [19] , AODV [20], DSR [21] or TORA [22]. The number of clusters in the network is determined by the extension that is to be covered by the whole network. If a new zone needs to be covered, a new cluster has to be added. Although many types of sensors or types of devices can be added to any cluster, the application of the $20 / 80$ rule (20\% of $\mathrm{CHs}, 80 \%$ of $\mathrm{CMs}$ ) is suggested [23]. 


\subsection{Identifiers and Predefined Parameters}

Base Station (BS) has the list of zone with an identifier called ZoneID and geographical coordinate of zone in network area. Each node deployed in the network with a unique 16-bit identifier called nodeID. When a node in a zone declared as cluster head by Base Station (BS) it acquires a unique 16-bit cluster head identifier called clusterID with zoneID in which it will work. Base station also declared a threshold value (TH). The selection of the new cluster head or for the $\mathrm{CH}$ election algorithm runs in the system when any of $\mathrm{CH}$ has its residual energy less or equal to threshold value. All nodes in a cluster will have the same cluster head and any new node will join the cluster whose $\mathrm{CH}$ is closest.

Every node in networks must have the following parameters in the proposed architecture:

- Position: position is the (x,y) geographical value for the node. It could be given manually or by GPS.

- Type: It identifies the type of node, whether it is $\mathrm{CH}$ or $\mathrm{CM}$.

With above parameter when a node is declared as $\mathrm{CH}$, following parameters are added by Base station to node.

- Max_con: Maximum number of supported connections from CMs.

- Max_distance: It is the maximum distance to be a neighbour. It is always shorter than or equal to the coverage area radius. The energy model as stated above specifies that distance can be calculated by signal strength. Maximum distance is the distance for transmission of request or information from the $\mathrm{CH}$.

Two parameters have been defined to be used for the operation of the architecture.

$\Delta$ Parameter: It depends on the node available energy and its age in the system. It is used to ascertain which node is the best one to become a Cluster Head node. A node with higher residual energy and the most stable node will be the CHs. Since the oldest node should be the lowest energy node, but this parameter appears to consolidate the most stable nodes as the CHs ( new ones could be mobile nodes or even with lower energy). A node with higher available energy and older will have higher $\Delta$. Equation 1 defines the $\Delta$ parameter:

$$
\Delta=16 \text {-age }\left(1-\mathrm{E}^{2}\right)^{1 / 2}
$$

where age $=\log 2($ nodeID $)$, so age varies from 0 to $16 . E$ is defined as $\%$ of energy consumption and it values vary from $0 \%$ to $100 \%$. $E=0$ indicates it is fully charged and $E=$ 100 indicates it is fully discharged.

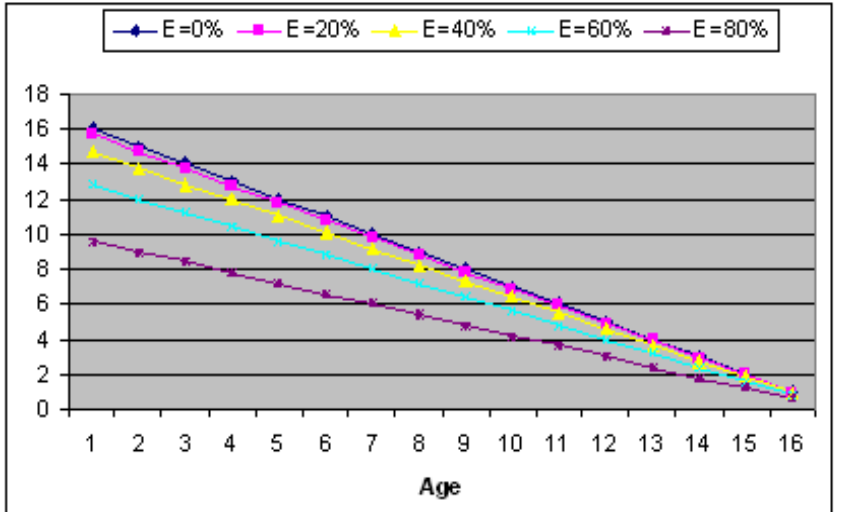

Figure 2: $\Delta$ values versus age 


\section{p Parameter}

It is the property of a node to become a cluster head. It is used by the Base Station or election algorithm to elect a node as $\mathrm{CH}$. When energy of any $\mathrm{CH}$ in the network is less then threshold value $\mathrm{CH}$ election algorithm will run in the system by the base station or by the existing $\mathrm{CH}$. $\mathrm{CH}$ election algorithm is run in the following scenario:-

1. For the first time when the network is deployed the election of $\mathrm{CH}$ is done by the BS.

2. When any of the existing cluster head is dead or non working with any reason then election algorithm is done by the base station.

3. When the $\Delta$ parameter of existing $\mathrm{CH}$ is equal to $\mathrm{TH}$ then election algorithm is run by the existing $\mathrm{CH}$ to select a new cluster head.

The $\rho$ parameter depends on the $\mathrm{CH}$ with the residual energy. If the residual energy of the $\mathrm{CH}$ $<=$ Threshold, value of $\boldsymbol{\rho}$ will be 0 . Value of $\boldsymbol{\rho}$ will be 0 or 10 , according to the state of the node. A 0 value of $\boldsymbol{\rho}$ indicates that the node is not elected as $\mathrm{CH}$ till now and a 10 value of $\boldsymbol{\rho}$ means it has the energy level at threshold value and not be elected till all node has the same $\boldsymbol{\rho}$ value. If all the nodes has same value of $\boldsymbol{\rho}(\boldsymbol{\rho}=\mathbf{1 0})$, than a node will be selected as cluster head with higher $\Delta$.

\section{SCALABILITY}

It is known that cluster based systems are more scalable than other systems. This section shows that why our proposal scales better than other proposals. First, we have to take into account that computation is much cheaper than communication in terms of energy dissipation [24]. So, what is desired is architecture with fewer retransmissions. This will imply a saving in energy of the whole system and it will give more scalability to the architecture.

Let a network of nodes $\mathrm{G}=(\mathrm{V}, \mathrm{E})$ be, where $\mathrm{V}$ is the set of nodes and $\mathrm{E}$ is the set of connections between nodes. Let $\mathrm{k}$ be a finite number of disjoint clusters of $\mathrm{V}$, so $\mathrm{V}=\mathrm{U}\left(\mathrm{V}_{\mathrm{k}}\right)$ and there is no node in two or more subsets $\left(\cap V_{k}=0\right)$, i.e., there are not overlapping nodes. Let us suppose $\mathrm{N}=\mid \mathrm{VI}$ (the number of nodes of $\mathrm{V}$ ) uniformly distributed in a region. Let us suppose that there is just one cluster head node per cluster, so there are $\mathrm{k}$ head clusters in the whole network. Equation 2 gives the number of nodes:

$$
N=\sum_{i=1}^{k}\left|V_{k}\right|
$$

and the average number of members of in a cluster will be given by Equation 3:

$$
\text { Average }=\frac{N}{k}
$$

By the architectural proposal and the above formula the average number of members in cluster is as in equation 3. The minimum number of neighbouring zone for a zone in network is 3 (If a zone is at corner of geographical area) and maximum of 8 neighbouring zone in the network (if it is in middle of the geographical area).

\section{ENERGY MODEL}

We assume that the energy consumption of the sensor is due to data transmission and reception. Cluster head is also consuming energy for the data aggregation before it sends the data to BS. We use the same radio model as stated in [13], [24] and shown in Figure 1, the energy consumed in transmitting one message of size $\boldsymbol{k}$ bits over a transmission distance $\boldsymbol{d}$, is given by

$\mathrm{E}_{\mathrm{Tx}}(\mathrm{k}, \mathrm{d})=\mathrm{k}\left(\mathrm{E}_{\text {elec }}+\Delta_{\mathrm{AMP}} \mathrm{d}^{\square}\right)=\mathrm{E}_{\text {elec }} \mathrm{k}+\mathrm{k} \Delta_{\mathrm{AMP}} \mathrm{d}^{\square}$ 
Where k=length of the message

$\mathrm{d}=$ transmission distance between transmitter and receiver

$E_{\text {elec }}=$ electronic energy

$\triangle_{A M P}=$ transmitter amplifier

$\Delta=$ Path Loss $(2<=\Delta<=4)$

Also, the energy consumed in the message reception is given by

$$
E_{r x}=E_{\text {elec }} k
$$

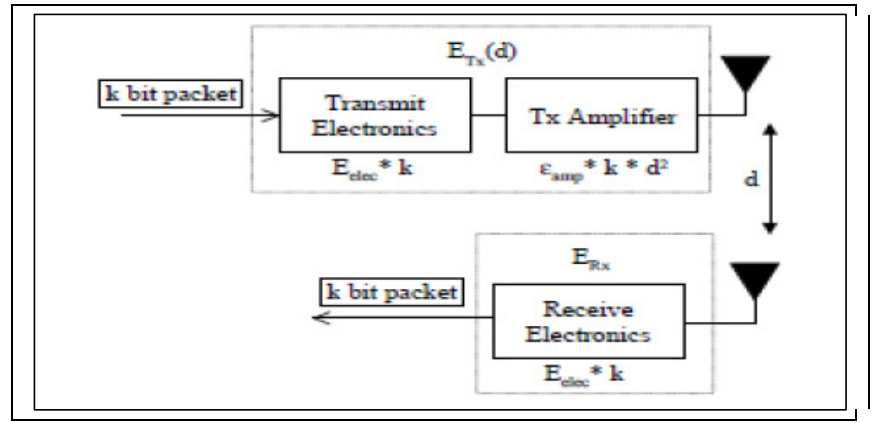

Figure3 : Radio Energy Model

\section{Proposed algorithm and Architecture Proposal Operation}

We proposed a new cluster head selection protocol for heterogeneous wireless sensor network. The algorithm is based on LEACH protocol. The proposed algorithm is zone-based algorithm and the parameter for heterogeneity is energy. In wireless sensor network two basic approaches are used to select the cluster head: Centralized and Distributed. In either of the method after a node is declared as a cluster head, the following process is followed to associate the node to cluster head:

1. The node declared as cluster head send the JOIN request to all other nodes.

2. The node receives the JOIN request and examines it on the basis of signal strength. According to general radio energy model for wireless sensor networks the signal strength depends on the distance means if signal comes from the more distance has the less strength and vice-versa.

3. A node will be selected as the cluster head, which has minimum distance from node.

4. Node sends a message for joining the cluster as cluster member.

The probability of the node to become a cluster head will be $\mathrm{P}, \mathrm{p}$ is the maximum number of cluster heads in the network. In the algorithm we reduce the number of communication between the sensor nodes for cluster head selection so that the energy consumption for cluster head selection can be further reduce. This can increase the residual energy of the cluster head and the network survivability can be enhanced. The emphasis of our approach is to increase the life span of the network by ensuring a uniform distribution of nodes in initial network. In the following section we describe the propose algorithm. Let us suppose a heterogeneous network with 100 nodes having different initial energy. The base station (BS) is located outside the deployed area and fixed. According to energy model data compression energy is different from the reception and transmission. Energy of transmission depends on the distance (source to destination) and data size. Although nodes are mobile but during the cluster head selection phase the nodes are assumed to be immobile.

The proposed algorithm works in round. Each round has the setup and transmission phase. 
The flow graph for the proposed algorithm is as follows :

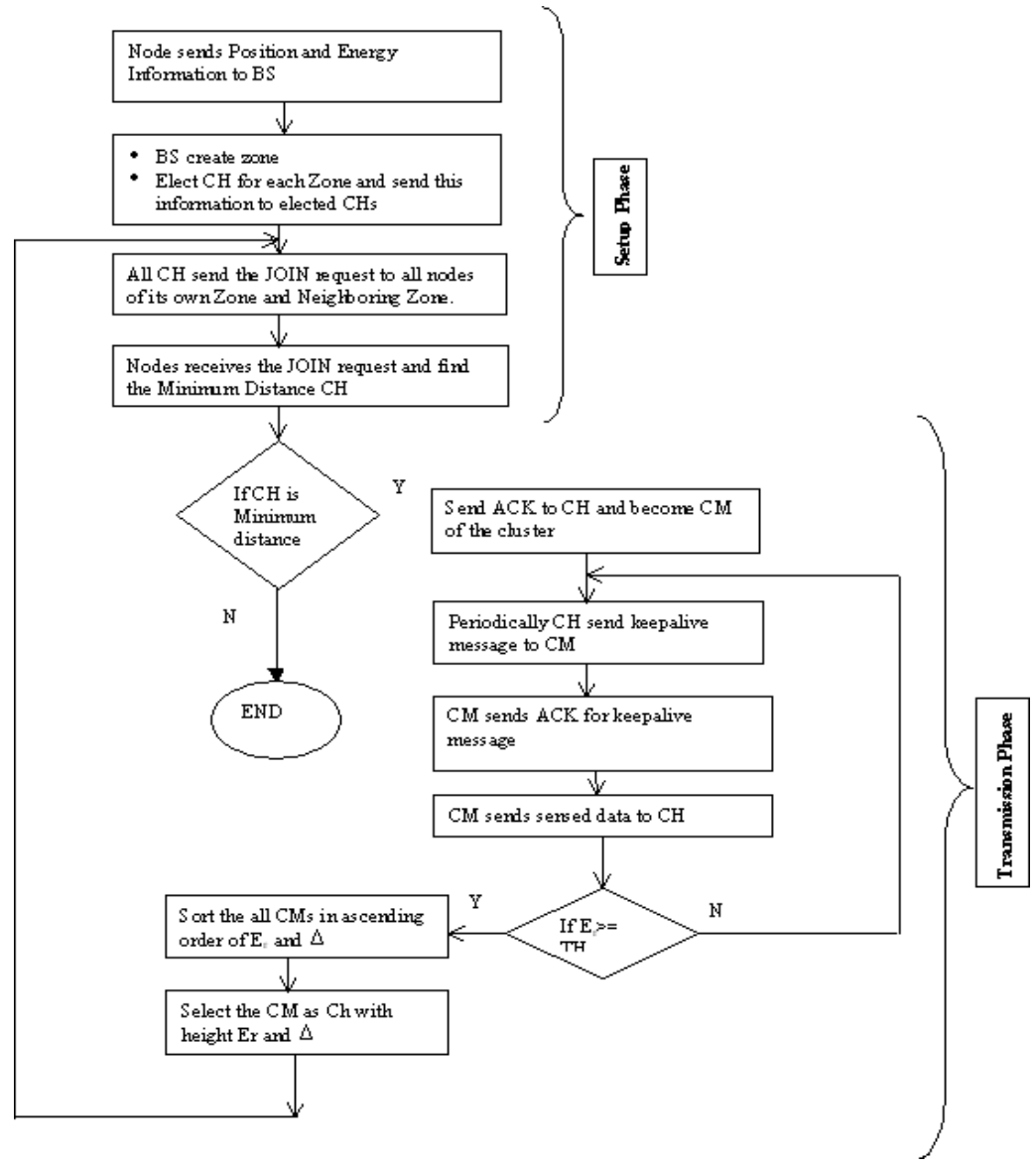

\subsection{Setup phase}

In the setup phase cluster head selection is done. Following steps are will be followed.

\subsubsection{Creating Zone}

- All nodes send the energy and position information to BS in SEND_INFO message.

- BS create zone on the basis of geographical area and assign a zoneID. For example we divide the network in 6 rectangles.

\begin{tabular}{|c|c|c|}
\hline $\begin{array}{lll}0 & 0 & \\
0 & 0 & 0 \\
0 & 0 & 0 \\
0 & 0\end{array}$ & $\begin{array}{|ccccc|} & & 0 & & \\
0 & 0 & 0 & 0 & 0 \\
0 & & 0 & \\
0 & 0 & 0 & 0 \\
0 & & & 0 & 0 \\
\end{array}$ & $\begin{array}{llll}0 & & 0 & 0 \\
0 & 0 & 0 & 0 \\
0 & 0 & 0 & 0 \\
0 & & 0 & 0 \\
0 & 0 & 0 & 0\end{array}$ \\
\hline 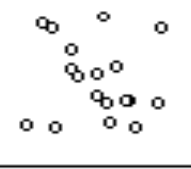 & $\begin{array}{ccccc}0 & 0 & 0 & 0 & 0 \\
0 & 0 & 0 & 0 \\
& 0 & & \\
0 & 0 & & 0 & 0 \\
0 & 0 & 0 & 0\end{array} \mid$ & 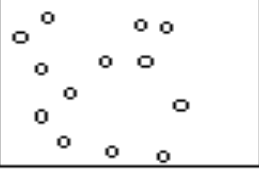 \\
\hline
\end{tabular}

Figure 4 : The sensor network

- By the position value it select node in zone and run cluster selection algorithm to select the cluster head. 
International Journal of Wireless \& Mobile Networks (IJWMN) Vol. 3, No. 3, June 2011

\begin{tabular}{|c|c|c|}
\hline \begin{tabular}{|cccc} 
& 0 & & \\
& 0 & 0 \\
& 0 & 0 \\
0 & 0 & 0 \\
& 0 & \\
\end{tabular} & 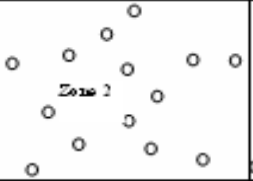 & 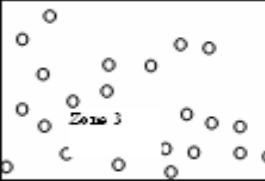 \\
\hline 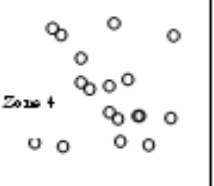 & 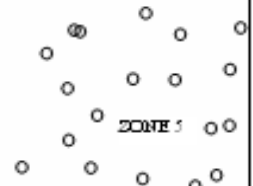 & 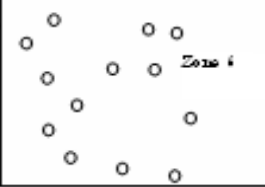 \\
\hline
\end{tabular}

Figure 5: Zone in the network.

- $\quad$ BS select $\mathrm{CH}$ elect Message node, elected as cluster head to cluster with clusterheadID and zoneID with which it associated by ELECT_INFO message.

\begin{tabular}{|c|c|c|}
\hline 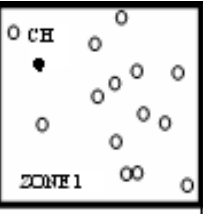 & 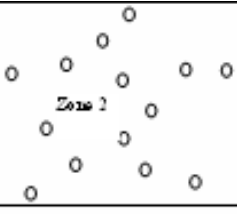 & 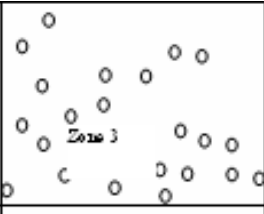 \\
\hline $\begin{array}{c}8000 \\
0 \\
8000 \\
800 \\
0000 \\
0.80\end{array}$ & 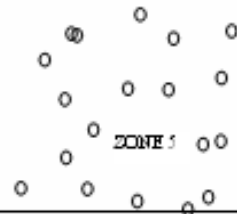 & $\begin{array}{cccc}0 & & 0 & 0 \\
0 & 0 & 0 & \text { Znew } \\
0 & & 0 \\
0 & 0 & 0 \\
0 & 0 & 0 \\
0 & 0 & 0\end{array}$ \\
\hline
\end{tabular}

Figure6 : Selection of Cluster Head in Zone 1

Protocol operation to create zone by BS:

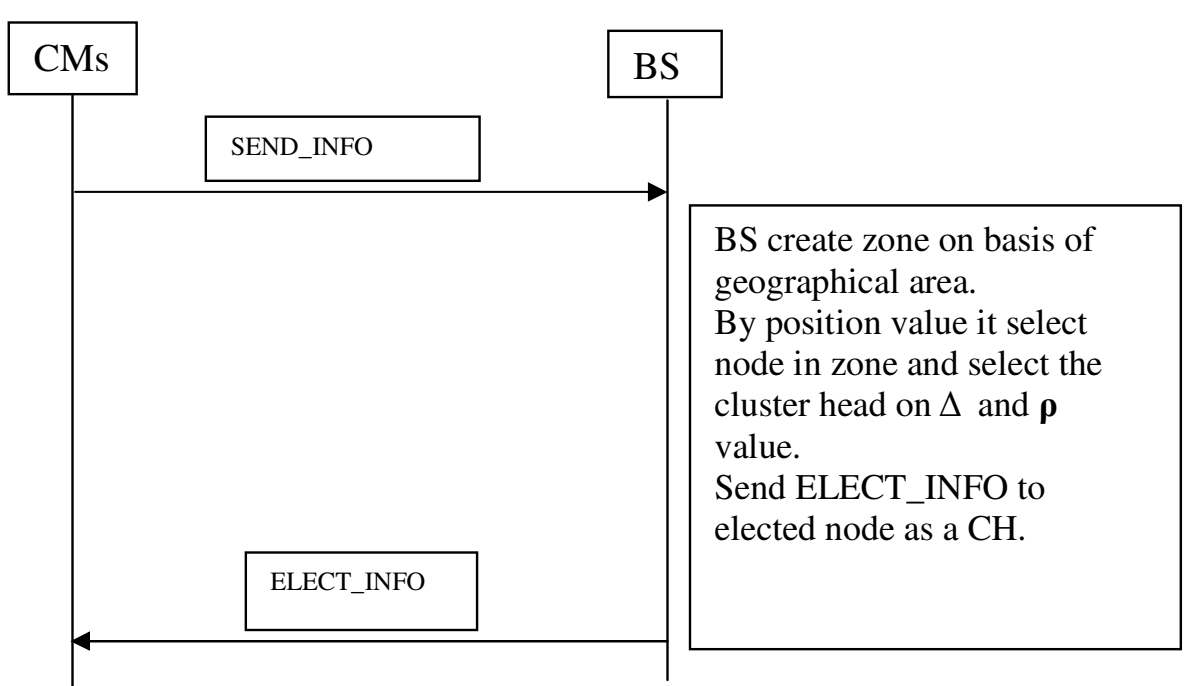

Figure 7 : Protocol operation to create zone and $\mathrm{CH}$ election by base station

In this process two messages are used :-

1. SEND_INFO (Energy and position information of Node)

2. ELECT_INFO.(ClusterID with ZoneID from which $\mathrm{CH}$ is associated). 
International Journal of Wireless \& Mobile Networks (IJWMN) Vol. 3, No. 3, June 2011

\subsubsection{Cluster Formation.}

- Selected cluster head send the JOIN request to the nodes lie in the zone itself and to the neighbour's zone. For example in figure the cluster head of Zone 1 will send the join request to the nodes of Zone 1, Zone 2, Zone4, and Zone 5.

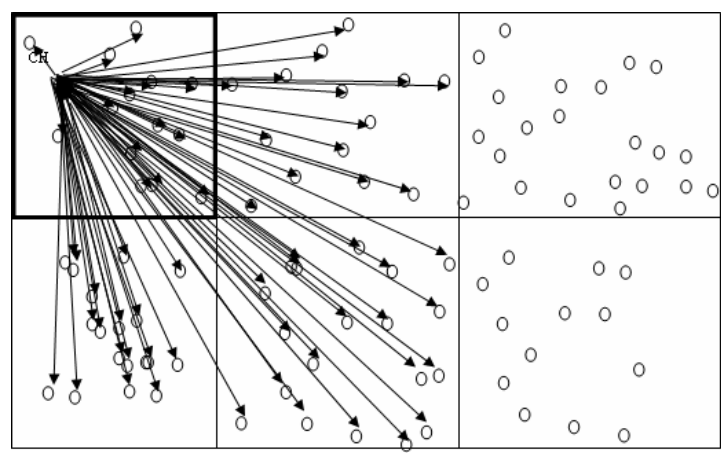

Figure 8 :Join request send to all other nodes in neighbouring zone

- Node receives all JOIN request and by their energy calculates the distance of the cluster heads.

- The node sends an ACK in response to the JOIN to the cluster head which has minimum distance.

\section{Protocol for cluster formation}

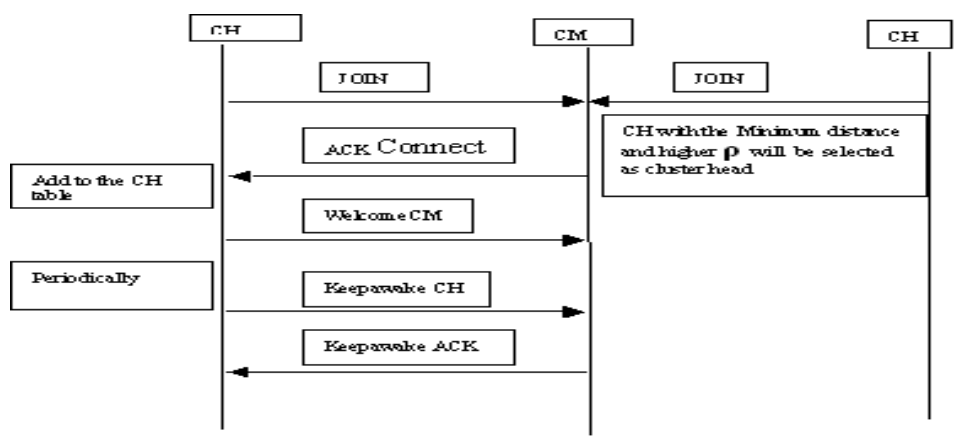

Figure 9 : Join request send to all other nodes in neighbouring Zone

- Final cluster formation is done.

- After welcome $\mathrm{CH}$ the cluster head periodically send the keepawake $\mathrm{CH}$ message to the node to check the whether the node is alive or not.

- The node responds this by keep awake ACK message to inform $\mathrm{CH}$ that it is in working condition. 


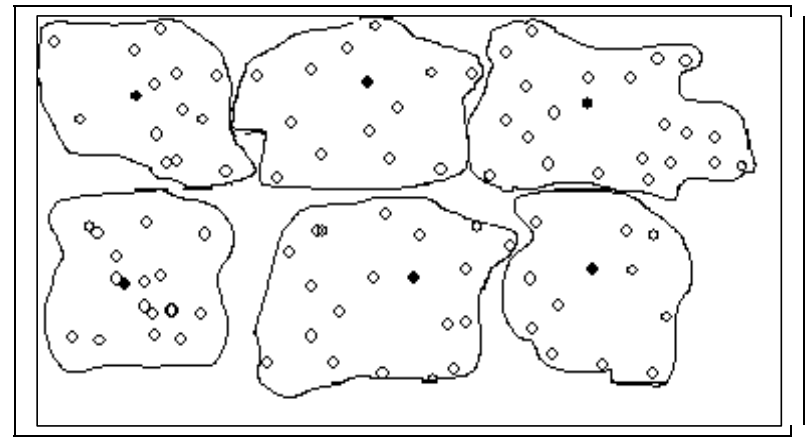

Figure 10 : Final cluster formation

The whole operation use the following Messages:

3. JOIN (cluster ID)

4. ACK connect(ClusterID, nodeID)

5. WelcomeCM(Cluster ID)

6. Keepawake(Beacon)

7. KeepawakeACK(Beacon)

\subsection{Transmission Phase}

1. Node sends data to cluster head in each round.

2. Cluster head receive the data, aggregate it and send it to the Base Station.

\subsection{NeW Cluster Formation}

New cluster is selected for the reasons described below

- When the energy of existing cluster heads decreases down to threshold value.

- When any of the existing cluster head leave the cluster, in case of failure or disconnection.

\subsubsection{When the energy of existing cluster heads decreases down to threshold value.}

In this situation the election algorithm is run by the existing cluster head. And after the election algorithm the cluster head will be the part of the network as normal node (CM).

- Nodes of the existing cluster are sorted in ascending order of energy.

- Node with the highest energy and with $\mathrm{R}$ value

- The hop distance from the existing cluster head is minimum (hop distance may 1 or 2).

- The new selected $\mathrm{CH}$ will again perform the step of setup phase to select to form a new cluster.

- Old CH send all the control information by Backup Message and cluster ID.

- New selected CH send the cluster ID to the BS with Zone ID with it nodeID in N_INFO message

- The new selected node send again send the JOIN request to all node in its own zone and to neighbour zone node.

- The node will select the clusterhead with minimum distance.

- With the ConnectACK a node can select the Cluster .

- Welcome $\mathrm{CH}$ is send by cluster head.

- And periodically it send the keepawake message to node which is responded by the keepawake ACK by node. 
- New Cluster with new $\mathrm{CH}$ will be created.

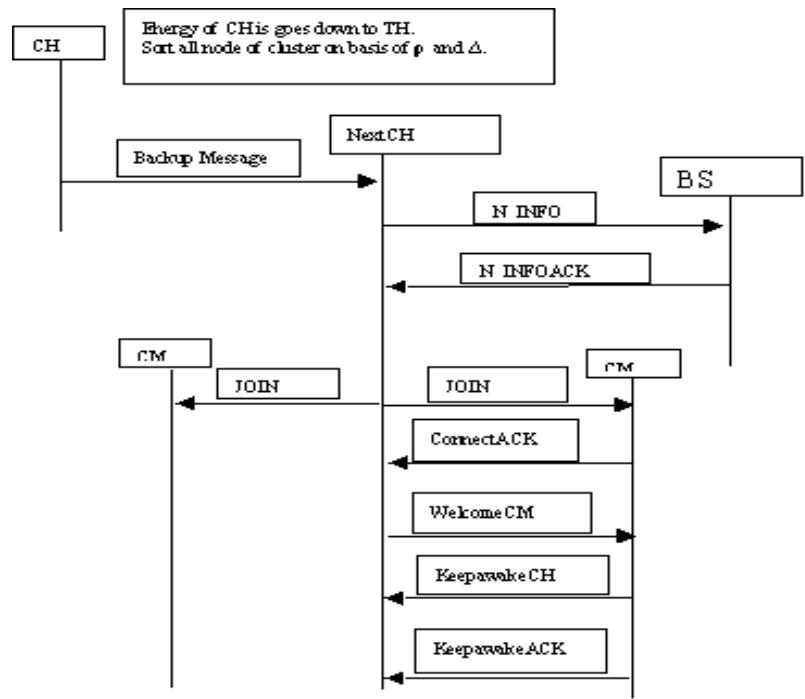

Figure 11 : Protocol operation for selecting new cluster head by existing cluster head

The whole process use the following new messages:-

8. Backup Message (nodeID,Cluser ID Associated with the $\mathrm{CH}$ )

9. N_INFO (Cluster ID, nodeID, zone ID)

10. N_INFOACK(ACK of n_INFO).

\subsubsection{New cluster head selection in case of Failure or disconnection.}

In this case the cluster head is not working in network due to failure or disconnection of $\mathrm{CH}$. This time the election algorithm is run by BS and it is same as describe in 6.1.

\subsection{ADDITION OF NEW NODE IN NETWORK}

- When a new node is added to the network it sends it geographical information to the BS.

- Then BS send it to the zoneID to the node.

- In order to join the architecture, the new node broadcasts a discovery message.

- CHs will reply with a discovery ACK message with their position .

Node will select the $\mathrm{CH}$ with following three ways.

6.4.1 If it does not receive any reply within 10 seconds, it becomes a $\mathrm{CH}$, so it creates the cluster and waits for new nodes. Ten seconds have been chosen because it is enough time to receive a reply from a near node. Later replies will be from nodes which are either too far or too busy. Then it send Discovery message to BS. BS station replies with ACK and associated zoneID and cluster ID of neighbouring zone. Then it send discovery message again to those node. If it receives one or more reply it follow the 6.4.3.

6.4.2 If it receives one or several replies and it finds the $\mathrm{CH}$ with minimum distance. Then, it sends an $\mathrm{M}$ connect message to establish a connection with the selected $\mathrm{CH}$. If the $\mathrm{CH}$ confirms that connection because it has not reached the maximum number of connections, it adds this entry to its $\mathrm{CM}$ table, sends a - Welcome $\mathrm{CH}$ message. 


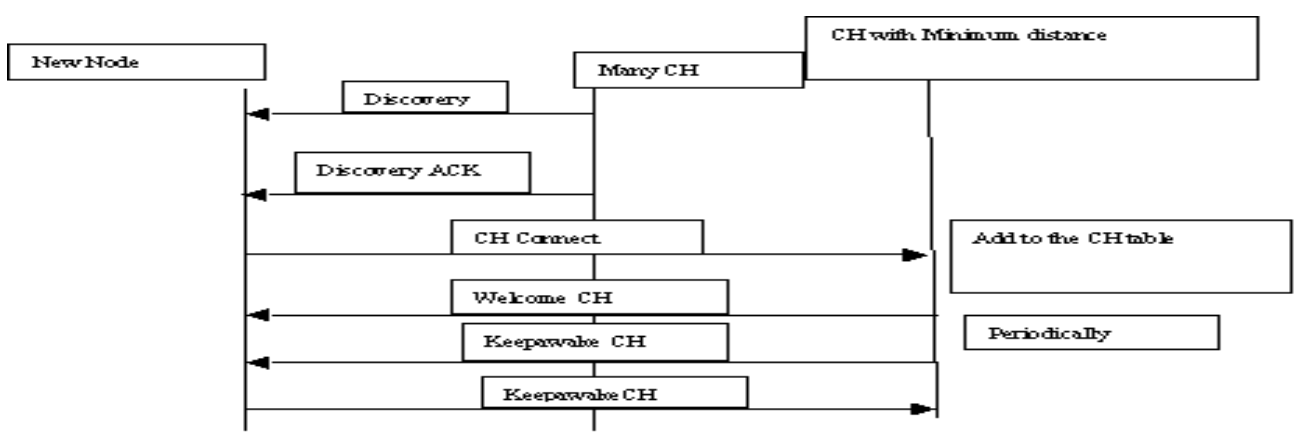

Figure 12: Protocol operation to select new cluster head by node

6.4.3 If it receives one or several replies and it finds the $\mathrm{CH}$ with minimum distance. Then, it sends an $\mathrm{M}$ connect message to establish a connection with the selected $\mathrm{CH}$. If the $\mathrm{CH}$ does not confirms that connection because it has reached the maximum number of connections, newly added node will send the M Connect message to second best $\mathrm{Ch}$ and follow the same steps.

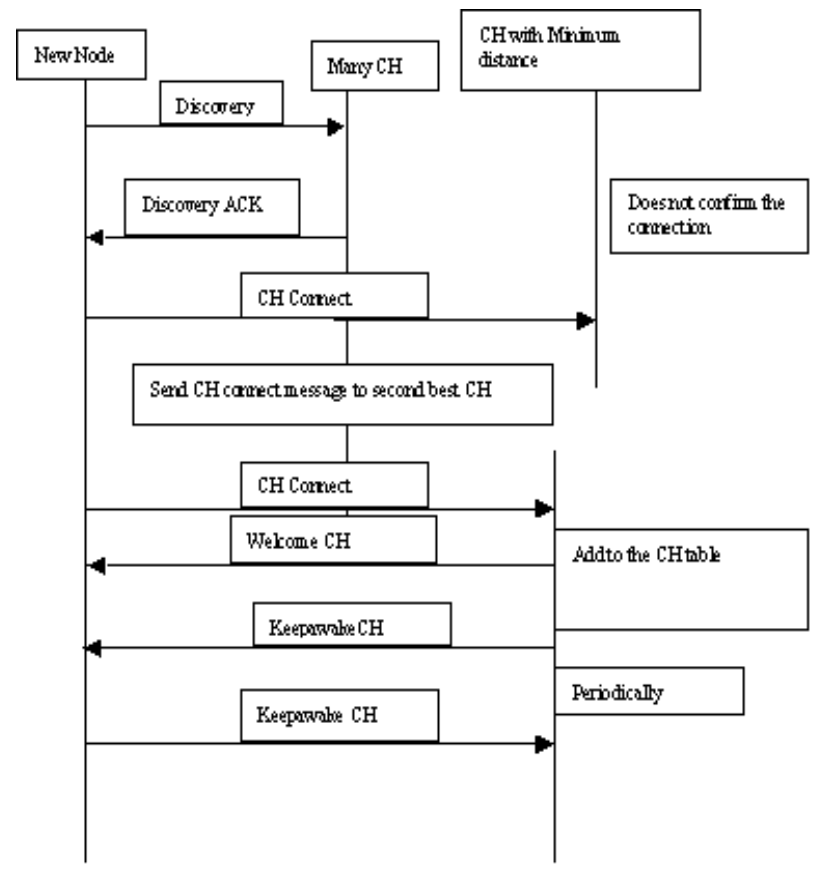

Figure 13 : Protocol operation to select new cluster head

The process uses the following message:

11. Discovery (nodeID)

12. DiscoveryACK(ClusterID, zone ID, nodeID).

\subsection{Deletion of node in network}

When a CM leaves the cluster (because of its mobility, or due to failure or other issues), the $\mathrm{CH}$ of its cluster will not receive any - keepawake message from it. After a dead time, the $\mathrm{CH}$ will erase this entry from its CM table. 


\subsection{Simulation}

Simulation result

The performance analysis of proposed clustering algorithm is evaluated in the MATLAB and compared with the LEACH algorithm (in which cluster selection is random selection process) in terms of the network lifetime. We simulate the proposed algorithm with the LEACH algorithm. In the LEACH random selection method in each round cluster head selection is on the basis of $1 /$ p. not on the residual energy.

The cluster head is determined by the following function (1)

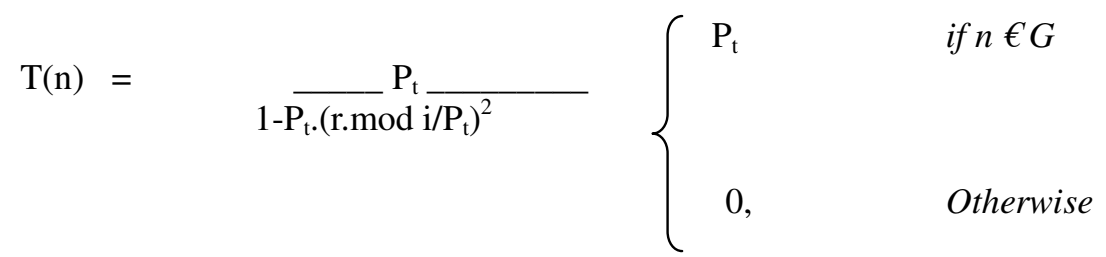

Where $\mathrm{Pt}$ is the desired percentage of cluster heads, $\mathrm{r}$ is the current round number; $\mathrm{G}$ is the set of nodes that have not been cluster-heads in the last $1 / \mathrm{Pt}$ rounds.

When a node is declared as cluster head it sends the JOIN request to all other nodes. In this simulation the node send the JOIN request to other 99 nodes but in the proposed algorithm the node has the maximum energy will be declared as cluster head and it send the JOIN request to the nodes of its own zone and neighbouring zone for example in the simulation if Zone 1, Zone 2, Zone 4, Zone5 have 72 nodes, then JOIN request will send to only 72 Nodes. Following energy can be saved 25 Transmission of JOIN request.

Power consumption in according to energy model method for cluster head selection and node association with the cluster head

$\mathrm{E}_{\text {consume }}=\mathrm{JOIN}$ request to all other nodes in network

+ Energy for the reception of data for all nodes in cluster

+ Send the $\mathrm{CH}$ information to Base station.

$=($ ETX $*$ ctrPacketLength + Emp* ctrPacketLength $)+($ ERX $*$ ctrPacketLength

$* \mathrm{~N})+\mathrm{EDA}+(\mathrm{ETX} *$ ctrPacketLength + Emp* ctrPacketLength $)$

Length of ctrPacketLength is 100 .

Table 1: Simulation of proposed and random algorithm

\begin{tabular}{|c|c|c|c|c|c|c|}
\hline & $\begin{array}{l}\text { Max no. o } \\
\text { cluster in } \\
\text { algorithm }\end{array}$ & $\begin{array}{l}\text { Maximum } \\
\text { number of nod } \\
\text { node in a In a cluster i } \\
\text { Proposed random } \\
\text { algorithm }\end{array}$ & $\begin{array}{l}\text { Number } \\
\text { JOIN } \\
\text { in request } \\
\text { Proposed } \\
\text { algorithm } \\
\end{array}$ & $\begin{array}{l}\text { of Number } \\
\text { JOIN } \\
\text { in request } \\
\text { Random } \\
\text { algorithm }\end{array}$ & \begin{tabular}{l|l|} 
Total \\
of Energy in \\
cluster \\
information \\
in random \\
algorithm \\
\end{tabular} & 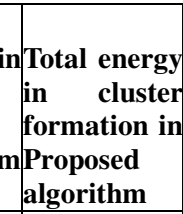 \\
\hline Simulation 1 & 25 & 50 & 76 & 99 & 75.0005 & 51.0005 \\
\hline Simulation 2 & 27 & 46 & 68 & 99 & 73.0005 & 48.0005 \\
\hline Simulation 3 & 24 & 38 & 65 & 99 & 69.0005 & 45.0005 \\
\hline Simulation 4 & 23 & 29 & 56 & 99 & 64.5005 & 40.0005 \\
\hline Simulation 5 & 21 & 37 & 69 & 99 & 68.5005 & 45.5005 \\
\hline Simulation 6 & 22 & 30 & 59 & 99 & 65.0005 & 41.0005 \\
\hline Simulation 7 & 27 & 32 & 63 & 99 & 66.0005 & 45.5005 \\
\hline \begin{tabular}{|l|} 
Simulation 8 \\
\end{tabular} & 24 & 39 & 66 & 99 & 69.5005 & 45.5005 \\
\hline Simulation 9 & 26 & 35 & 72 & 99 & 67.5005 & 49.5005 \\
\hline Simulation 10 & 24 & 42 & 68 & 99 & 71.0005 & 46.5005 \\
\hline
\end{tabular}


International Journal of Wireless \& Mobile Networks (IJWMN) Vol. 3, No. 3, June 2011

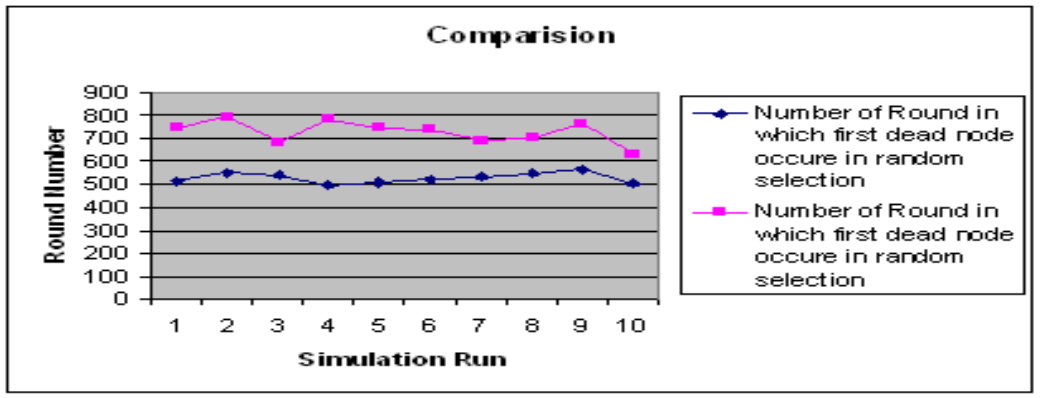

Figure 14 : Comparision operation to select new cluster head



Figure 15 : Network Stability

\section{Protocol Comparision}

Table 2 : Comparision of various protocols

\begin{tabular}{|l|l|l|l|l|l|l|l|}
\hline $\begin{array}{l}\text { Clustering } \\
\text { Algorithm }\end{array}$ & $\begin{array}{l}\text { Energy } \\
\text { Efficient }\end{array}$ & $\begin{array}{l}\text { Location } \\
\text { awareness }\end{array}$ & $\begin{array}{l}\text { Balanced } \\
\text { clustering }\end{array}$ & $\begin{array}{l}\text { Cluster } \\
\text { stability }\end{array}$ & $\begin{array}{l}\text { Heterogeneity } \\
\text { Type }\end{array}$ & $\begin{array}{l}\text { Clustering } \\
\text { Methodology }\end{array}$ & $\begin{array}{l}\text { Heterogen } \\
\text { eity Level }\end{array}$ \\
\hline $\begin{array}{l}\text { Kumar } \\
\text { 2009 }\end{array}$ & High & No & Yes & Moderate & Energy & Distributed & Three \\
\hline Qing2006 & High & No & Yes & Moderate & Energy & Distributed & Two \\
\hline $\begin{array}{l}\text { Elbhiri } \\
\mathbf{2 0 0 9}\end{array}$ & High & No & Yes & Good & Energy & Distributed & Two \\
\hline Duan 2007 & High & No & Yes & Good & Energy & Distributed & Two \\
\hline $\begin{array}{l}\text { Marin- } \\
\text { Perianu } \\
\mathbf{2 0 0 8}\end{array}$ & High & Yes & No & Moderate & $\begin{array}{l}\text { Energy and } \\
\text { Link }\end{array}$ & Centralized & Multilevel \\
\hline $\begin{array}{l}\text { Smaragda } \\
\text { kis 2004 }\end{array}$ & Low & No & & Good & Energy & Distributed & Two \\
\hline $\begin{array}{l}\text { Haibo } \\
\text { Larma }\end{array}$ & Low & Yes & Yes & $\begin{array}{l}\text { Very } \\
\text { Good }\end{array}$ & $\begin{array}{l}\text { Computational } \\
\text { and Energy }\end{array}$ & Centralized & Three \\
\hline Li 2007 & Low & Yes & Yes & Good & $\begin{array}{l}\text { Computational } \\
\text { and Energy }\end{array}$ & Centralized & Two \\
\hline $\begin{array}{l}\text { Wang } \\
\mathbf{2 0 0 7}\end{array}$ & Ok & No & Yes & $\begin{array}{l}\text { Very } \\
\text { Good }\end{array}$ & Energy & Distributed & Two \\
\hline Liaw 2009 & Low & No & Yes & Good & Centralized & Two \\
\hline $\begin{array}{l}\text { PECP } \\
\text { High }\end{array}$ & Yes & Yes & Good & Energy & Semi & Multilevel. \\
\hline
\end{tabular}




\section{Conclusion}

The wireless sensor networks have been envisioned to help in numerous monitoring applications. Energy efficient routing is paramount to extend the stability and lifetime of the system. In this paper, we have proposed an energy efficient heterogeneous clustered scheme for wireless sensor networks. The energy efficiency and ease of deployment make PECP a desirable and robust protocol for wireless sensor networks. In order to improve the lifetime and performance of the network system, this paper reports on the development of an architecture that creates clusters based on zone and establishes connections between sensor nodes and also the description of the protocol developed and the flow of the messages have been presented. Comparing our proposal with others, it can be seen that the detailed description of our protocol allows its easy implementation Simulations results show that PECP has extended the lifetime of the network as compared with LEACH in the presence of same setting of powerful nodes in a network. Hence, the performance of the proposed system is better in terms of reliability and lifetime. Although we compared PECP with LEACH, there are many clustering algorithms that we have to compare and there are many factors that can affect the network lifetime. Further directions of this study will be deal with clustered sensor networks with more than two levels of hierarchy.

\section{REFERENCES}

1 Charles E. Perkins. “Ad Hoc Networking” Addison-Wesley, Boston, MA, first edition, 2001.

2. N. Al-Karaki and A. E. Kamal. "Routing techniques in wireless sensor networks: a survey". In IEEE Wireless Communications, Volume 11, pp. 6- 28, 2004.

3. Yick, J.; Mukherjee, B.; Dipak, D. Wireless Sensor Network Survey. Comput. Netw. 2008, 52, 2292-2330.

4. Krco, S. Health Care Sensor Networks-Architecture and Protocols. Ad Hoc. Sensor Wireless Networks 2005, 1, 1-25.

5. Mainwaring, A.; Szewczyk, R.; Anderson, J.; Polastre, J. Habitat Monitoring on Great Duck Island.In Proceedings of ACM SenSys'04, Baltimore, MD, USA, November 2004.

6. Summers, S.A. Wireless Sensor Networks for Firefighting and Fire Investigation; CS526 Project. UCCS: Colorado Springs, CO, USA, 2006.

7. Yang, H.; Sikdar, B. A Protocol for Tracking Mobile Targets Using Sensor Networks. In Proceedings of SNPA'03, Anchorage, AK, USA, May 2003; pp. 71-81.

8. Jiang, M.; Li, J.; Tay, Y.C. Cluster Based Routing Protocol (CBRP). August 1998. Available online: http://tools.ietf.org/html/draft-ietf-manet-cbrp-spec-01.txt (accessed on 3 December 2009).

9. Yu, J.Y.; Chong, P.H.J. A Survey of Clustering Schemes for Mobile Ad Hoc Networks. IEEE Commun. Surv. Tutorials 2005, 7, 32- 48.

10. Lin, C.R.; Gerla, M. Adaptive Clustering for Mobile Wireless Networks. IEEE J. Sel. Areas Commun. 1997, 15, 1265-1275.

11. Ryu, J.H.; Song, S.; Cho, D.H. New Clustering Schemes for Energy Conservation in Two- Tiered Mobile Ad Hoc Networks. In Proceedings of IEEE ICC'01, Helsinki, Finland, June 2001; pp. 862866.

12. Abbasi, A.A.; Younis, M. A Survey on Clustering Algorithms for Wireless Sensor Networks.Comput. Netw. 2007, 30, 2826-2841.

13. W. R. Heinzelman, A. Chandrakasan, and H. Balakrishnan. "Energy efficient communication 
International Journal of Wireless \& Mobile Networks (IJWMN) Vol. 3, No. 3, June 2011

protocol for wireless microsensor networks". In Proceedings of the Hawaii International Conference on System Sciences, 2000.

14. S. Lindsey, C.S. Raghavendra, "PEGASIS: power efficient gathering in sensor information systems", in: Proceedings of the IEEE Aerospace Conference, Big Sky, Montana, March 2002.

15. Dilip Kumar a,*, Trilok C. Aseri b,1, R.B. Patel "EEHC: Energy efficient heterogeneous clustered scheme for wireless sensor networks in "Computer Communications" 32 (2009) 662-667.

16. Vivek Katiyar, Narottam Chand, Surender Soni ," Clustering Algorithms for Heterogeneous Wireless Sensor Network: A Survey “,International Journal Of Applied Engineering Research, Dindigul Volume 1, No 2, 2010

17. Changmin Duan; Hong Fan,"A Distributed Energy Balance Clustering Protocol for Heterogeneous Wireless Sensor Networks", Wireless Communications, Networking and Mobile Computing, 2007.

18. R.S. Marin-Perianu $\square$, J. Scholten, P.J.M. Havinga And P.H. Hartel Cluster -based service discovery for heterogeneous wireless sensor networks International Journal of Parallel, Emergent and Distributed Systems, Vol. , No. , , 1-35.

19. Clausen, T.; Jacquet, P. Optimized Link State Routing Protocol (OLSR). RFC 3626. October 2003. Available online: http://www.ietf.org/rfc/rfc3626.txt (accessed on 3 December 2009).

20. Perkins, C.; Belding-Royer, E.; Das, S. Ad Hoc On-Demand Distance Vector (AODV) Routing. RFC 3561. July 2003. Available online: http://www.ietf.org/rfc/rfc3561.txt (accessed on 3 December 2009).

21. Johnson, D.; Hu, Y.; Maltz, D. The Dynamic Source Routing Protocol (DSR) for Mobile Ad hoc Networks for IPv4. RFC 4728. February, 2007. Available online: http://www.ietf.org/ rfc/rfc4728.txt (accessed on 3 December 2009).

22. Park, V.; Corson, S. Temporally-Ordered Routing Algorithm (TORA) Version 1, Functional Specification, Internet Draft. June 2001. Available online: http://tools.ietf.org/id/draft-ietf-manettora-spec-04.txt (accessed on 3 December 2009).

23 Chang, Y,C.; Lin, Z.S.; Chen, J.L. Cluster Based Self-Organization Management Protocols for Wireless Sensor Networks. IEEE Trans. Consum. Electron. 2006, 52, 75-80.

24. Heinzelman, W.R.; Chandrakasan, A.; Balakrishnan, H. Energy-efficient communication Protocol for Wireless Microsensor Networks. In Proceedings of the 33rd Annual Hawaii International Conference on System Sciences, Maui, HI, USA, January 2000; Volume 2.

25. Smaragdakis, G., Matta, I. and Bestavros, A. (2004). SEP: A stable election protocol for clustered heterogeneous wireless sensor networks, In: Proc. of the International Workshop on

SANPA 2004. pp 251261

26. Haibo, Z., Yuanming, W., Yanqi, H. and Guangzhong, X. (In Press) A novel stable selection and reliable transmission protocol for clustered heterogeneous wireless sensor networks, Computer Communications, In Press, Corrected Proof.

27. Varma, S., Nigam, N. and Tiwary, U.S. (2008). Base station initiated dynamic routing protocol for Heterogeneous Wireless Sensor Network using clustering, Wireless communication and Sensor Networks, WCSN 2008, pp 16.

28. Li, X., Huang, D. and Sun, Z. (2007). A Routing Protocol for Balancing Energy Consumption in Heterogeneous Wireless Sensor Networks, MSN 2007, LNCS 4864: pp 79-88..

29. Li, X., Huang, D. and Yang, J. (2007). Energy Efficient Routing Protocol Based on Residual Energy and Energy Consumption Rate for Heterogeneous Wireless Sensor Networks, In: The 26th Chinese Control Conference, 5: pp 587-590.

30. Wang, X. and Zhang, G. (2007). DECP: A Distributed Election Clustering Protocol for Heterogeneous Wireless Sensor Networks, LNCS; 4489: Proceedings of the 7th international conference on Computational Science, pp 105108. 
International Journal of Wireless \& Mobile Networks (IJWMN) Vol. 3, No. 3, June 2011

31. Liaw, J.J., D., ChenYi and YiJie, W. (2009). The Steady Clustering Scheme for Heterogeneous Wireless Sensor Networks, Ubiquitous, Autonomic and Trusted Computing, UICATC '09. Symposia and Workshops on, pp 336341.

Authors

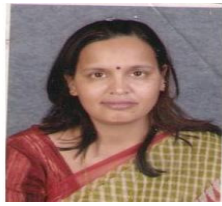

S.Taruna is an active researcher in the filed of communication and mobile network, currently working as Assistant Professor in Department of Computer Science at Banasthali University ( Rajasthan), India. She has done M.Sc from Rajasthan University and her $\mathrm{PhD}$ is in progress from Banasthali University(Rajasthan), India.

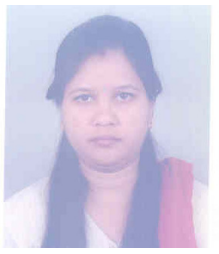

Kusum Lata Jain has done M.sc from Banasthali University and currently pursuing M.Tech in Computer Science from Banasthali University (Rajasthan), India. She has interest in the field of communication network.

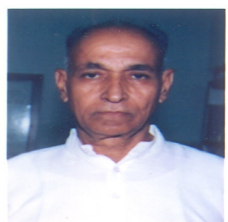

Prof. G. N. Purohit is a Professor in Department of Mathematics \& Statistics at Banasthali University (Rajasthan). Before joining Banasthali University, he was Professor and Head of the Department of Mathematics, University of Rajasthan, Jaipur. He had been Chief-editor of a research journal and regular reviewer of many journals. His present interest is in O.R., Discrete Mathematics and Communication networks. He has published around 40 research papers in various journals. 